\title{
Simulated nature walks improve psychological well-being along a natural to urban continuum
}

\author{
Giuliana Brancato ${ }^{1}$, Kathryne Van Hedger ${ }^{2,3,4}$, Marc G. Berman ${ }^{5}$, Stephen C. Van Hedger¹,2,6 \\ ${ }^{1}$ Department of Psychology, Huron University College at Western, London, Canada \\ ${ }^{2}$ Brain and Mind Institute, University of Western Ontario, London, Canada \\ ${ }^{3}$ BrainsCAN, University of Western Ontario, London, Canada \\ ${ }^{4}$ Department of Clinical Neurological Sciences, University of Western Ontario, London, Canada \\ ${ }^{5}$ Department of Psychology, University of Chicago, Chicago, IL, United States \\ ${ }^{6}$ Department of Psychology, University of Western Ontario, London, Canada
}

\section{Author Note:}

Number of Tables: 1

Number of Figures: 4

Abstract Word Count: 266

Word Count (main body): 6,792

${ }^{*}$ Corresponding Author: Department of Psychology, Huron University College at Western, 1349 Western Road, London, ON N6G 1H3 Canada: svanhedg@uwo.ca

The authors declare no conflicting interests. All data associated with this paper are available through Open Science Framework (https://osf.io/xgkvn/) 


\begin{abstract}
Compared to urban environments, interactions with natural environments have been associated with several health benefits including psychological restoration and improved emotional wellbeing. However, classifying environments dichotomously as either natural or urban may emphasize between-category differences and minimize potentially important within-category variation (e.g., forests versus fields of crops; neighborhoods versus city centers). Therefore, the current experiment assessed how viewing brief videos of different environments, ranging along a continuum from stereotypically natural to stereotypically urban, influenced subjective ratings of mood, restoration, and well-being. Participants were randomly assigned to one of four video conditions, which depicted a simulated walk through a pine forest, a farmed field, a tree-lined urban neighborhood, or a bustling city center essentially devoid of greenery. Immediately before and after the videos, participants rated their current emotional states. Participants additionally rated the perceived restorativeness of the video. The results supported the idea that the virtual walks differentially influenced affect and perceived restoration, even when belonging to the same nominal category of natural or urban. The pine forest walk significantly improved happiness relative to both urban walks, whereas the farmed field walk did not. The bustling city center walk decreased feelings of calmness compared to all other walks, including the tree-lined neighborhood walk. The walks also differed on two perceived restorativeness measures (daydreaming and being away) in a graded fashion; however, the farmed field walk was found to be less fascinating than all other walks, including both urban walks. Taken together, these results suggest that categorizing environments as "natural versus urban" may gloss over meaningful within-category variability regarding the restorative potential of different physical environments.
\end{abstract}

Keywords: Nature, Attention Restoration Theory, Affect, Attention, Categorization 


\section{Simulated nature walks improve psychological well-being along a natural to urban continuum}

Interactions with nature have been associated with psychological benefits, including improved emotional well-being and cognitive functioning. Immersion into the natural world offers a salve for our mental resources, increasing positive affect and decreasing negative affect (Felsten, 2009). A considerable body of research suggests connecting with natural environments promotes well-being and positive feelings (Capaldi et al., 2014, 2015; Martin et al., 2020; McAllister et al., 2017; McMahan \& Estes, 2015), and points to a buffering effect between exposure to nature and the adverse effects caused by mental fatigue (Brymer et al., 2010). Moreover, multiple experimental studies have shown that exposure to natural environments can improve affect (e.g., Bratman et al., 2015) and performance on tasks requiring attention and working memory (e.g., Berman et al., 2008; Stenfors et al., 2019; Van Hedger, Nusbaum, Clohisy, et al., 2019) compared to similar exposure to urban settings. Several theories offer explanations for these consistent affective and cognitive enhancements of natural environments.

The Biophilia Hypothesis (Kellert \& Wilson, 1993; Wilson, 1984) and Stress Reduction Theory (SRT; Ulrich et al., 1991) offer explanations for the affective benefits of interacting with nature. Rooted in an evolutionary perspective, the Biophilia Hypothesis (Kellert \& Wilson, 1993; Wilson, 1984) suggests that humans have a strong affiliation with other living things because throughout most of human history people existed outdoors as members of hunter-gatherer societies. The number of plant and animal species in a given area, known as biodiversity, might signal the presence of significant survival resources, creating a positive association between biodiversity and thriving life (Wood et al., 2018). Similarly, SRT posits that the aesthetic and affective attribution of nature is sufficient to promote parasympathetic autonomic activity and as a result reduces unpleasant feelings of stress (Ulrich, 1981, 1983, 1984; Ulrich et al., 1991). SRT more specifically states that the aesthetic evaluation of natural resources such as vegetation and water rapidly (and perhaps automatically) elicit these physiological changes, presumably due to evolutionarily salient cues such as the availability of survival-based resources (Ulrich, 1983). Yet, these theories have been supported by research comparing stereotypically natural environments to urban environments that are largely devoid of naturally inhabiting plants and animals (e.g., Ulrich et al., 1991). Some recent research has found results that may be somewhat at odds with these theories. First, a recent paper found that children actually prefer urban environments compared to natural environments, and over time develop preferences for nature (Meidenbauer et al., 2019). Furthermore, other research has shown that 
when natural and urban environmental stimuli are equated on preference, that nature stimuli do not improve mood any more than equally preferred urban stimuli (Meidenbauer et al., 2020). Both of these recent results suggest that the positive effects of natural environments on mood are likely to be more nuanced than suggested by either the biophilia hypothesis or SRT.

Attention Restoration Theory (ART; Kaplan, 1995), suggests time spent in nature allows for cognitive resources to become restored, resulting in temporarily improved attentional abilities when re-entering urban life - which in turn can have benefits for psychological well-being. For cognitive resources to become restored, Kaplan (1995) outlined a set of conditions that need to be met by the environment. The environment must foster feelings of: (1) being away, (2) extent, (3) fascination, and (4) compatibility. First, being away allows for a distance between oneself and mentally fatiguing situations that are common in our day to day lives. Notably, the theory points out this need not be a physical distance; for example, virtual exposure to the natural world (e.g., Brown et al., 2013; McAllister et al., 2017) can still elicit mental distance between an individual and their stressors. Second, extent is the degree to which an environment is expansive and can be explored broadly. Here too, extent does not mean that the environment needs to be spatially expansive, though that is one way to increase extent. Small spaces, such as Japanese gardens or labyrinths are often able to provide feelings extent even though the environments may not be large spatially. Third, compatibility is described as the degree to which an individual's environment fits with their goals and provides the necessary information to meet those goals. Fourth, fascination refers to interesting or captivating situations that effortlessly capture attention. Other researchers have also suggested that the perceptual features of nature, such as the melodic contours of birdsong, are thought to be "softly fascinating," allowing individuals to address lingering or unresolved thoughts that would otherwise become mentally draining (Basu et al., 2019). Without periods of reflection, cognitive resources become fatigued, contributing to an overall decrease of psychological well-being (Basu et al., 2019; Kaplan, 1995; Kaplan \& Berman, 2010; McMahan \& Estes, 2015; Wood et al., 2018). Thus, stereotypically natural environments (e.g., forests) often meet these four conditions and are thus considered excellent environments for attention restoration. However, researchers have not yet fully examined the extent to which non-stereotypical natural and urban environments might also offer some degree of restoration (cf. White et al., 2010).

Urban environments are often juxtaposed to natural environments in studies of the benefits of nature. Prior studies of urban environments have shown that these environments tend to deplete mental and attentional resources relative to natural environments (for review see Kaplan \& Berman, 2010; Schertz \& Berman, 2019; Stenfors et al., 2019; Stevenson et al., 
2018), leaving little opportunity for reflection and increased cognitive load. In many research studies urban spaces are operationalized as devoid of meaningful greenery, despite the fact that such greenery is common within city neighborhoods in the form of trees or parks interspersed among built features like buildings and roads. Intriguingly, a study by Felston (2009) found that real exposure to urban green space landscapes was not associated with mental restoration. In contrast, a number of studies have found psychological benefits of urban green space (Dadvand et al., 2015; Engemann et al., 2019; Kardan et al., 2015; Schertz et al., $2018,2021)$, suggesting that the relationship between urban green space and psychological well-being may be nuanced and depend on the specific context of the greenery (e.g., perceived richness of biodiversity; Southon et al., 2018; Wilkie et al., 2020; Wyles et al., 2019). Specific efforts to categorize environments as either "natural" or "urban" can exacerbate the differences between these two types of environments.

Categorization can be useful when understanding the effects of an environment on affect and cognition, but it also minimizes potentially important intra-natural and intra-urban variability. Indeed, findings from research assessing the interactions between nature and well-being oversimplify the potential differences between natural environments. In fact, researchers have claimed that this dichotomy may be overly simplified, as Kaplan \& Berman (2010) theorized that while many natural environments may restore directed attention, these are not the only environments that could do so and in fact there may be urban environments that could also improve directed attention if those environments did not tax directed attention, while simultaneously having stimulation that might capture involuntary attention softly (Berman et al., 2019; Kaplan \& Berman, 2010).

Studies often operationalize nature in terms of "green space," defined as the ratio of greenery in a given area. Although green space is an effective way to quantify nature, there is a risk of treating diverse ecosystems as equivalent. For example, one cubic meter in a grassy field and one cubic meter in a rainforest are both considered "green space," yet these environments are distinctive in both perceptual features and biodiversity. Green space is common within city neighborhoods in the form of parks and tree-lined streets. Wood and colleagues (2018) assessed 12 parks with varying levels of biodiversity (e.g., number of plant and bird species, habitat diversity), yet all the parks were comparable in terms of their green space (i.e., percentage of tree cover). Participants walking through each of the 12 parks were asked to take part in a survey assessing the restorative benefits from their nature walk. The findings suggest that the restorative effects of nature exposure could be predicted by the level of biodiversity in the park, even after controlling for age, gender, and ethnic background. As a result, the 
literature quantifying nature using green space appears to be insufficient, and more recent investigations have suggested that considering the content of green space is critical in explaining the restorative potential of both canonically natural and urban environments (Southon et al., 2018; Wilkie et al., 2020). Kardan and colleagues (2015) also found a specific effect of trees on health and maybe even a slightly stronger effect for evergreens compared to deciduous trees. Schertz and colleagues (2021) found that tree canopy was a significant predictor of reduced crime, but that grass coverage was not, again, suggesting that all green spaces are not created equally.

In this study we sought to address the question of how different natural and urban environments might be differentially associated with psychological well-being and restoration of mental resources. This is an important question as the results have the potential to offer insight into features of natural and urban environments that account for the well-documented positive benefits of natural environments and ways to mitigate the potential negative effects of urban environments. To better understand the effects of different natural and urban environments on psychological well-being and perceived restoration, the current experiment includes virtual walks through four conditions: a high biodiversity nature setting (pine forest); a low biodiversity nature setting (farmed field); an urban setting with considerable greenery (tree-lined city neighborhood); and an urban setting devoid of greenery (bustling city center). We hypothesized that psychological well-being would be positively impacted along a continuum from canonical natural to canonical urban environments (i.e., forest to farmed field to tree-lined city neighborhood to bustling city center). We also hypothesized that perceived restorativeness would be similarly impacted along the same continuum.

\section{Method}

\section{Participants}

202 participants were recruited using Amazon Mechanical Turk, an online recruitment platform. The sample size was determined by an a priori power analysis. Using G*Power (Faul et al., 2009) we calculated that a sample size of 180 participants would provide sufficient statistical power (.80) for detecting differences in a between-participant one-way ANOVA, assuming a medium effect size. We recruited more than 180 participants in anticipation of excluding some participants due to low-quality or incomplete responses. Cloud Research (Litman et al., 2017) was used to further constrain participant recruitment from Mechanical Turk. Specifically, only participants who passed attention and data quality checks implemented by Cloud Research were eligible to participate. In order to enroll in the study, participants were 
required to be fluent in English (to read and answer the survey questions) and had to have a high-speed internet connection (for loading a virtual walk video). Headphone use was encouraged but was not required. The research protocol was approved by the Huron Research Ethics Board.

\section{Procedure}

Participants were provided with a letter of information, which specified the details of the study, and provided their informed consent before beginning the study. Those who consented were randomly assigned to one of four conditions. The four conditions were characterized by different virtual walk environments (pine forest, farmed field, tree-lined city neighborhood, bustling city center). Participants were instructed to find a quiet place in which they felt comfortable to turn on computer sound or to use headphones; participants were also reminded to ensure their volume was at an appropriate level before continuing. Next, participants rated their current mood. Then, participants completed a 15-minute virtual walk. At the end of the virtual walk, participants were given a unique code to input into the survey to confirm that they had watched the entire video. Immediately after entering their unique completion code, participants completed the same mood measure that had been administered prior to the virtual walk. Next, participants completed state measures of affect and anxiety, and rated the restorativeness of the virtual walk, in a randomized order. Following the state measures and restorativeness scales, participants completed trait measures of personality, anxiety, loneliness, and relatedness to nature, again in a randomized order. After completing the trait measures, participants completed a short demographic questionnaire. Finally, participants were asked to briefly describe what they saw and heard in the video in a free-response question. This was intended as an attention check to verify that participants had watched the video as instructed. Participants were then thanked for their participation, given monetary compensation for their time, and received a debriefing form containing the purpose and hypotheses for the study. Figure 1 summarizes the experimental design.

\section{Materials}

The virtual walk videos (see Supplemental Information for links and green space analyses) were selected from YouTube using the following keywords: first-person forest walk, first-person field walk, and first-person urban walk. Figure 2 provides representative screenshot images from each video. The original videos ranged from 16 to 88 minutes in duration; however, only the first 15 minutes of each video were shown to participants. All videos used a first-person 
perspective. The pine forest walk was recorded on a nature trail along the Metolius River in Deschutes National Forest in Oregon. The farmed field walk did not contain any tags with respect to a specific location; however, it depicted a walk on a farm through a green, grassy field. The tree-lined neighborhood walk was recorded in the Beacon Hill neighborhood of Boston, MA. The bustling city walk was recorded in New York city, specifically through Times Square and midtown Manhattan. In terms of greenery, the two nature videos were statistically comparable and were both had significantly more greenery than both urban videos (see Figure SI1 and SI2). The videos were trimmed, and presented using jsPsych, an open-source JavaScript library for conducting psychological research in a web browser (de Leeuw, 2015).

Participants also completed current state questionnaire measures of mood, affect, and anxiety, and two measures assessing the perceived restorativeness of the walk video after it was viewed. Finally, four questionnaires assessed participants' trait characteristics, with respect to personality, anxiety, loneliness, and relatedness to nature. These measures were not hypothesized to change as a function of video condition but were collected to ensure that participants were well-matched across conditions. All questionnaire measures were administered using Qualtrics.

\section{State Measures}

Participants completed visual analogue scale (VAS) mood measures immediately before and after watching the virtual walk video. The VAS asked participants to rate how they were feeling at that moment on five terms (happy, sad, calm, anxious, and lonely) using a 100-point slider scale with higher ratings indicating a greater extent of feeling that term.

The state component of the State-Trait Anxiety Inventory for Adults (STAI-S; Spielberger, 1983) was used to assess participants' current feelings of anxiety after the virtual walk video. The STAI-S consisted of 20 items (e.g., "I feel calm", "I feel nervous"). Participants were asked to rate each item on a four-point Likert scale from 1 (not at all) to 4 (very much so).

Similar to the STAI-S, the Positive Affect Negative Affect Schedule (PANAS; Watson et al., 1988) was used to measure affect after the virtual walk video. The PANAS contains 10 positive affect (e.g., "proud") and 10 negative affect (e.g., "guilty") words that describe feelings and participants are asked to rate the extent of their current feelings on a five-point Likert scale from 1 (not at all) to 5 (extremely).

\section{Restorativeness Scales}


The Mental Bandwidth Scale (MBS; Basu et al., 2019) is a seven-item scale designed to assess mental activities such as reflection and self-awareness during activities - in this case, the virtual walk. The scale contains three subcomponents. First, self-awareness addresses participants' awareness of their surroundings and internal thoughts (e.g., "during this video, I was able to take note of thoughts and feelings"). Second, daydreaming is meant to assess mind-wandering (e.g., "during this video, to what degree were you lost in thought?"). Third, planning assesses the extent to which, during the virtual walk, participants were lost in thought for events in the past or future (e.g., "during this video, to what degree were you making plans for the future?"). Participants rated each item on a five-point Likert scale from 1 (not at all) to 5 (extremely).

The Perceived Restorativeness Scale (PRS; Norling et al., 2008) is a nine-item scale meant to assess the therapeutic potential of environments, explicitly focused on activities - in this case, the virtual walk. Similar to the MBS, the PRS consists of three subcomponents. First, being away focuses on capturing the extent to which participants feel removed from the taxing demands of day-to-day life (e.g., "this activity is an escape for me"). Second, fascination addresses the attentional effort required for the task (e.g., "this activity has many fascinating qualities"). Third, extent captures the level of effort required to engage in the activity (e.g., "this activity sustains my interest"). Each item was rated by participants on a five-point item Likert scale from 1 (not at all) to 5 (extremely) after viewing the virtual walk.

\section{Trait Measures}

The Ten Item Personality Inventory (TIPI; Gosling et al., 2003) was used to assess the Big Five personality traits: (1) openness to experience, (2) conscientiousness, (3) extraversion, (4) agreeableness, and (5) emotional stability. Each item consists of a word pair (e.g., "extroverted, enthusiastic"), and participants rated the extent to which the words generally described them using a seven-point Likert scale, from 1 (disagree strongly) to 7 (agree strongly).

The trait component of the State-Trait Anxiety Inventory for adults (STAI-T; Spielberger, 1983) was administered to assess participants' trait levels of anxiety (i.e., independent of the virtual walk manipulation). Participants were instructed to rate 20 statements (e.g., "I lack confidence") based on how they feel generally. The state and trait portions of the state-trait anxiety inventory for adults were administered as two separate questionnaires. The response scale was identical to the STAI-S. 
The short-form UCLA Loneliness Scale (ULS-8; Hughes et al., 2004) was used to assess participants' general feelings of loneliness in everyday life. Participants rated eight items (e.g., "I feel left out") on a 5-point Likert scale, ranging from 1 (not at all) to 5 (extremely).

Lastly, the Nature Relatedness Scale (NRS; Nisbet et al., 2009) assesses an individual's general level of connectedness to the natural world. Participants were asked to rate 21 items (e.g., "my relationship to nature is an important part of who I am") on a 5-point Likert scale, ranging from 1 (not at all) to 5 (extremely).

\section{Data Analysis and Exclusion Criteria}

In a preliminary analysis, participants were removed from the data analysis if they completed the study in fewer than 20 minutes, were missing more than one answer on any questionnaire, or did not pass the attention check. To pass the attention check, participants had to describe what they experienced in the first-person walk in detail. The authors (GB, SVH) were able to reach a consensus in terms of what constituted sufficient detail. In total, of the 202 initial participants, 24 participants were removed based on these considerations, leaving a total of 178 participants in the primary analysis (Pine Forest: $n=45$, Farmed Field: $n=48$, Tree-lined Neighborhood: $n=41$, Bustling City: $n=44)$. The mean age of the included participants was $40.67(S D=11.93$, range of 21 to 72 years old).

A one-way analysis of variance (ANOVA) was used to assess differences in the scales as a function of walk type (pine forest, farmed field, tree-lined neighborhood, bustling city), with walk type being a between-participant factor. As the VAS was the only scale administered both before and after the intervention, the analysis of the VAS used a 2x3 ANOVA, with time (preintervention, post-intervention) as a within-participant factor and walk type (pine forest, farmed field, tree-lined neighborhood, bustling city) as a between-participant factor. Given the relatively large number of administered measures and subsequent ANOVAs, all analyses were subject to False Discovery Rate (FDR) multiple comparison corrections (Benjamini \& Hochberg, 1995), implemented in R. As such, all primary results report FDR-corrected $p$-values ( $q$-values). If the $q$-value for a particular measure was significant, we ran post-hoc tests, which used BonferroniHolm corrections for multiple comparisons.

\section{Results}

\section{Visual Analogue Scale}

The VAS results are plotted in Figure 3. Given that the VAS was the only measure that was administered before and after the virtual walk, we first assessed whether there were 
baseline differences among the walk conditions. Although there were nominal differences among the walk conditions, no analysis of pretest score was significant (all qs > .210). For happiness ratings there was a main effect of time, $F(1,174)=25.39, q=.007, \eta^{2} p=.127$, with happiness ratings significantly increasing after watching the virtual walk. We also observed a significant interaction of time and video condition, $F(3,174)=7.54, q=.007, \eta^{2} p=.115$. This interaction was characterized by a significantly greater increases in happiness ratings for the pine forest video compared to both urban videos (both $p s=.003$ ). The increase in happiness observed in the farmed field video condition did not significantly differ from any of the other conditions $(p>$.242), and the two urban videos did not significantly differ from one another $(p=$ $.976)$. The main effect of condition was not significant, $F(3,174)=1.06, q=.507, \eta^{2}{ }_{p}=.018$.

Sadness ratings did not show a significant main effect of time, $F(1,174)=2.44, q=.278$, $\eta^{2} \mathrm{p}=.014$, nor a significant interaction of time and condition, $F(3,174)=1.79, q=.294, \eta^{2} p=$ .030. There was also no main effect of condition for sadness ratings, $F(1,174)=1.49, q=.352$, $\eta^{2} \mathrm{p}=.025$. Given the nonsignificant findings, no post-hoc analyses were performed.

Calmness ratings showed a significant main effect of time, $F(1,174)=8.75, q=.025, \eta^{2} p$ $=.048$, with calmness ratings significantly increasing after the virtual walk. Time also interacted with condition, $F(3,174)=6.36, q=.007, \eta^{2} p=.099$, suggesting that the relative increase in calmness ratings differed as a function of walk type. Specifically, the interaction was driven by the bustling city video, which showed a decrease in calmness ratings post-video and was significantly different from all other video conditions (all ps <.009). No other video conditions significantly differed from one another (all ps $=1$ ). The main effect of condition was not significant, $F(3,174)=1.49, q=.352, \eta^{2} p=.025$.

Anxious ratings showed a significant main effect of time, $F(1,174)=13.10, q=.007, \eta^{2} p$ $=.070$, with anxiousness significantly decreasing after the virtual walk. Time did not significantly interact with condition, $F(3,174)=3.30, q=.102, \eta^{2} p=.054$. Although the bustling city walk nominally increased anxious ratings, whereas all other walks nominally decreased anxious ratings, this interaction did not survive FDR corrections. The main effect of condition was also not significant, $F(3,174)=1.70, q=.298, \eta^{2}{ }_{p}=.028$.

Loneliness ratings did not show a main effect of time, $F(1,174)=2.98, q=.238, \eta_{p}^{2}=$ .017 , with loneliness nominally (but non-significantly) decreasing after the virtual walk. There was no significant interaction between time and condition, $F(3,174)=0.48, q=.784, \eta^{2} p=.008$. There was also no significant main effect of condition, $F(3,174)=0.23, q=.878, \eta^{2} p=.004$.

\section{Mental Bandwidth Scale}


The Daydreaming subscale of the MBS showed a significant effect of virtual walk condition, $F(3,174)=6.36, q=.007, \eta_{p}^{2}=.099$. The pattern of results (Figure 4) suggests that daydreaming was influenced in a graded fashion and was the highest for the pine forest walk and lowest for the bustling city walk. Post-hoc tests showed that both the pine forest and farmed field walks significantly differed from the bustling city walk ( $p<.001$ and $p=.028$, respectively). Additionally, the difference between the pine forest and tree-lined neighborhood walks was marginally significant $(p=.080)$. All other comparisons were nonsignificant ( $p s>.293$ ).

The Self-Awareness subscale of the MBS did not significantly differ across virtual walk conditions, $F(3,174)=0.59, q=.720, \eta^{2} p=.010$. Mean scores for the pine forest, farmed field, tree-lined neighborhood, and bustling city conditions were $2.98(S D=0.75), 2.90(S D=0.70)$, $2.83(S D=0.81)$, and $3.03(S D=0.82)$, respectively.

The Planning subscale of the MBS also did not significantly differ across virtual walk conditions, $F(3,174)=0.83, q=.612, \eta^{2} p=.014$. Mean scores for the pine forest, farmed field, tree-lined neighborhood, and bustling city conditions were $1.86(S D=0.89), 1.78(S D=0.93)$, $1.60(S D=0.61)$, and $1.67(S D=0.80)$, respectively.

\section{Perceived Restorativeness Scale}

The Being Away subscale of the PRS did not differ as a function of walk type, $F(3,174)$ $=2.60, q=.210, \eta_{p}^{2}=.043$. Similar to the Daydreaming subscale of the MBS, the pattern across conditions was characterized by a graded decrease as a function of green space, with the pine forest walk eliciting the greatest sense of being away and the busting city walk eliciting the lowest sense of being away (Figure 4). However, it is important to note that this did not survive the FDR correction and thus should not be meaningfully interpreted.

The Fascination subscale of the PRS significantly differed as a function of walk type, $F(3,174)=4.09, q=.042, \eta_{p}^{2}=.066$. Unlike the MBS Daydreaming subscale, the Fascination subscale (Figure 4) did not show a graded effect as a function of green space. Rather, post-hoc tests showed that the farmed field walk was rated as significantly lower in fascination compared to the pine forest $(p=.021)$, and bustling city $(p=.016)$, and marginally lower in fascination compared to the tree-lined neighborhood $(p=.070)$.

The Extent subscale of the PRS did not differ as a function of walk type, $F(3,174)=0.66$, $q=.686, \eta^{2} \mathrm{p}=.011$. The mean extent scores were $3.50(S D=1.19), 3.24(S D=1.20), 3.41(S D$ $=1.16)$, and $3.20(S D=1.04)$ for the pine forest, farmed field, tree-lined neighborhood, and bustling city walks, respectively. 


\section{Positive Affect Negative Affect Schedule}

The positive affect items of the PANAS did not significantly differ across virtual walk conditions, $F(3,174)=1.33, q=.408, \eta^{2} p=.022$. The mean scores for the positive affect items of the PANAS were $2.92(S D=0.92), 3.10(S D=0.72), 2.89(S D=0.71)$, and $3.18(S D=0.81)$ for the pine forest, farmed field, tree-lined neighborhood, and bustling city walks, respectively.

The negative affect items of the PANAS also did not significantly differ across virtual walk conditions, $F(3,174)=2.44, q=.210, \eta^{2} p=.040$. The mean scores for the negative affect items of the PANAS were $1.31(S D=0.52), 1.24(S D=0.53), 1.10(S D=0.17)$, and $1.37(S D=$ 0.57 ) for the pine forest, farmed field, tree-lined neighborhood, and bustling city walks, respectively.

\section{State Anxiety}

The state measure of the STAI did not significantly differ as a function of virtual walk condition, $F(3,174)=1.75, q=.294, \eta^{2} \mathrm{p}=.029$. The mean state anxiety scores were $1.66(S D=$ $0.58), 1.65(S D=0.57), 1.46(S D=0.32)$, and $1.70(S D=0.62)$ for the pine forest, farmed field, tree-lined neighborhood, and bustling city walks, respectively.

\section{Trait Measures}

None of the trait measures (Table 1) differed across the virtual walk conditions, suggesting that the participants were comparable in terms of personality, trait anxiety, loneliness, and general feelings of connectedness with nature.

\section{Discussion}

The current study was designed to assess whether brief, virtual walks through different natural and urban environments would influence measures of psychological well-being and restoration. We found clear evidence that the walks differentially influenced both affect and perceived restoration in a manner that could not be entirely predicted by nominal category (i.e., "natural" versus "urban") or by the amount of green space in each environment. As such, our results highlight the potential benefits of virtual walks for improved well-being and restoration. However, our results also suggest that common heuristics to determine the "naturalness" of an environment may not always provide the most appropriate means of representing restorative potential.

In terms of affect, we found that the walks had differing effects on both positive and negative aspects of mood by examining change scores from before and after the virtual walks. 
All of the walks nominally increased participants' ratings of happiness, but the pine forest walk led to greater increases in happiness compared to both walks through urban environments (tree-lined neighborhood and bustling city). The bustling city walk made participants feel less calm than before the walk, while all of the other walks, including the tree-lined neighborhood walk, made participants feel more calm and less anxious than before the walk. Interestingly, however, the virtual walks did not substantially change ratings of sadness or loneliness. These results demonstrate that strict dichotomous categorization of walks into "natural" and "urban" can occlude the potential mood enhancing benefits of some urban environments (e.g., tree-lined neighborhood), while also overestimating the benefits of others (e.g., the farmed field walk did not increase happiness ratings to the same extent as the pine forest walk). Despite some of the nuances observed across walk conditions, these affective results are generally consistent with the principles of SRT. Although the pine forest and farmed field were comparable in terms of green space, the greenery and natural elements in both videos were qualitatively different. In the pine forest, participants experienced wild vegetation and running water, which may rapidly confer affective changes due to the evolutionary significance of these features signaling thriving life (Ulrich, 1981; Ulrich et al., 1991). In contrast, the greenery contained within the farmed field walk was relatively homogenous and "tame" (e.g., containing clearly visible lines between rows of planted crops) and therefore may not have conferred the same affective responses in participants. As such, these results suggest that both the type and quality (i.e., biodiversity) of an environment's green space contributes to its potential for engendering affective changes.

Although the findings from the VAS are consistent with SRT, an alternative explanation is that participants' culturally developed preferences for particular environments influenced their affective responses. In support of these findings, Meidenbauer et al. (2019) found that the preference for nature develops gradually, with children actually preferring urban environments. Meiderbauer et al. (2020) additionally found that other highly preferred images can engender similar affective benefits as nature. In the auditory domain, listeners do not inherently prefer the acoustic features that are representative of nature sounds (Van Hedger, Nusbaum, Heald, et al., 2019). As such, the present findings are also consistent with a cultural developmental view, in which preferences for nature emerge more gradually and confer affective benefits due to this developed preference. One potential way of disentangling these theories would be to replicate the current experiment in children, who might not have strong explicit preferences for nature (Meidenbauer et al., 2019). 
Given the walk-related changes in positive affect (e.g., happiness and calmness) measured by the VAS, it is perhaps surprising that we did not observe a similar effect of walk condition on the positive affect component of the PANAS. This could be due to a couple of reasons. First, the PANAS includes several terms in its positive affect dimension that may not be relevant to a virtual walk intervention (e.g., "proud" or "strong"). Second, the PANAS was not administered as a repeated measure, meaning it might have been less sensitive to changes brought about by the different walk conditions. In contrast, the negative affect results were more consistent across measures, in the sense that they had a generally weaker (or non-significant) relationship with the virtual walk. Specifically, we did not observe a significant effect of walk condition on sadness, anxiousness, or loneliness on the VAS, we did not observe a significant effect of walk condition on the negative affect component of the PANAS, and we did not observe a significant effect of walk condition on the state measure of the STAI. Thus, our results generally suggest that virtual walks through different green space environments might have a stronger tendency to increase positive affect rather than decrease negative affect. This is consistent with prior studies (e.g., Meidenbauer et al., 2020).

The virtual walks in this study also had some effects on perceived restorativeness, in line with predictions grounded in ART. Both the pine forest and farmed field walks encouraged daydreaming more than the bustling city walk. Given that daydreaming stems from internally generated thought and is facilitated by the availability of higher levels of mental bandwidth (Basu et al., 2019; McMillan et al., 2013), these findings are conceptually aligned with prior studies that have used more stereotypical natural vs. urban categorizations (e.g., Berman et al., 2012). However, it is also notable that the tree-lined neighborhood walk did not significantly differ from either nature walk in terms of the degree to which it encouraged daydreaming. The walks also nominally differed on feelings of "being away" in a graded fashion, with the pine forest walk eliciting the greatest sense of being away and the busting city walk eliciting the lowest sense of being away. This is aligns with findings by Wilkie and colleagues (2020) that 'being away' was not commonly mentioned on social media in reference to urban green space environments.

Despite being "natural" and containing significant green space, the farmed field walk was rated lowest on the fascination component of the Perceived Restorativeness Scale, significantly lower than the pine forest walk. These results are consistent with Attention Restoration Theory (Kaplan, 1995) as a pine forest walk contains sufficient biodiversity to softly capture attention and is consistent with prior associations between nature and ART. In contrast, the farmed field walk was visually consistent and somewhat mundane, despite still taking place in a natural (i.e., 
not man-made) setting. This result suggests an environment's restorativeness cannot be entirely predicted based on whether it is considered natural and urban and that not all natural environments softly capture attention, just like not all urban environments harshly capture attention. Notably, the pine forest walk and the farmed field walk were well matched on green space, but differed on content (e.g., biodiversity) and perceptual features (e.g., curved edges) that might contribute to the cognitive benefits of natural environments above and beyond the mere presence of green space.

It is additionally interesting to note that the bustling city walk was rated comparably to the pine forest walk in terms of fascination. Fascination is considered an integral component of a restorative environment (cf. Kaplan, 1995), and thus this finding was unexpected. However, one explanation for this pattern of results is that participants in the bustling city walk condition were actually rating "hard fascination" (i.e., situations in which directed attention is strongly engaged) rather than "soft fascination" (i.e., situations in which involuntary attention is captured, allowing directed attention to replenish). This potential interpretation is supported by the relatively low ratings of daydreaming in the bustling city walk condition. Thus, future research should consider administering both the PRS and MBS, as was done in the present study, to help clarify participant interpretations of restorative qualities. These findings could also be clarified with performance-based measures of attention or working-memory.

Exposure to nature is often reported to promote feelings of restoration, both psychologically and physically (Kaplan, 1995; McMahan \& Estes, 2015; Ulrich et al., 1991) and the current experiment is consistent with previous research in which exposure to nature improved psychological well-being. The present study demonstrates that virtual exposure to nature (via a simulated walk) is sufficient to impact psychological well-being, which suggests that virtual walks may constitute a promising intervention for psychological restoration outside of experimental contexts. Prior studies comparing in vivo exposure and virtual exposure have reported that virtual exposure is associated only with moderate improvement in positive affect (McMahan \& Estes, 2015). However, these studies have demonstrated that virtual exposure to nature is associated with decreases in negative affect comparable to real exposure. McMahan and Estes (2015) compared nature photographs of tamed (urban green space) versus wild (high vegetation preserves) landscapes. Virtual exposure to wild natural landscapes showed a more significant restorative effect than similar exposure to tamed landscapes, and surprisingly, the real exposure (nature walk) from Study 1 and wild virtual exposure had the same effect on wellbeing. This suggests virtual exposure that mimics a high level of natural resources such as water and vegetation (e.g., the pine forest walk in the present study) may act as a replacement 
for real exposure, at least in terms of perceived psychological restoration. One explanation for why exposure to virtual environments can also impact psychological well-being could be due to the fact that virtual environments preserve low-level perceptual features that exist in real world environments.

In the present study, the pine forest walk elicited the strongest positive impact on psychological well-being and restorativeness, but the tree-lined neighborhood walk had effects that were more similar to the two "natural" videos than to the other "urban" video, particularly with respect to the pre-post mood assessments. Berman et al. (2014) noted that visual features like non-straight edges (e.g., contours of tree branches) may be associated with soft fascination and reflection, and the presence of these features might be key in eliciting the beneficial effects from interactions with natural environments. From these results, it seems that relying on the "urban" vs. "natural" distinction often ignores the extent to which these features can also be present in urban environments. Moreover, we found that the farmed field walk was rated lower than all other walks in terms of the "fascination" component of restorativeness - this again raises the question as to how different features of both natural and urban environments might be responsible for different dimensions of restorativeness. Indeed, there is nothing exclusive about specific features (e.g., curved lines) and nature, as exemplified by emerging research in biophilic architectural design (i.e., build environments that mimic natural features; see Asim et al., 2020; Coburn et al., 2019). The findings from this study suggest that caution should be exercised in concluding that all possible environments may be categorized as either fitting within a natural or an urban framework, as this kind of categorization can minimize important withincategory differences. The results of the current study, consistent with the findings from Wood et al. (2018), point to the need to recharacterize "natural" beyond a single category.

The current study has a few limitations. First, while the use of a virtual walks allows for the integration of both audio and visual experiences, virtual exposure to different environments cannot capture the complex multi-sensory aspects of real-world interactions. Second, only two natural environments were assessed. Given the ecological and geographic diversity in the natural world, these results cannot be generalized to natural environments outside of those assessed in the current study (e.g., rainforest, beach, snow-capped mountains); however, this is an important area of exploration for future research. Third, the present experiment was administered online. Although online research is not inherently limited relative to laboratorybased research, the present experiment required participants to passively watch a 15-minute video without any direct prompting or attention checks during the video. Without controlling the participants' surrounding environment as they watched the video, the degree to which the 
simulated walk was "immersive" cannot be known. Even though the participants were instructed to find a quiet place to complete the study, controlling the background environment is not possible in an online experimental context. Fourth, the present study did not include any performance-based measures of cognitive restoration. A recent review suggests that performance-based measures may be more stringent than self-report measures in assessing the true restorativeness of natural environments (Browning et al., 2021). Despite these limitations the current findings offer a compelling example of the benefits virtual walks can have for improving psychological well-being and offer some interesting avenues for future research to assess within- and between-category differences when comparing natural and urban environments. Furthermore, the fact that the present study was conducted entirely online suggests that videos of virtual walks may represent a promising intervention for improving psychological well-being, even outside of experimental contexts.

\section{Conclusion}

The current study was designed to test the effects of different virtual walks through natural and urban environments on psychological well-being and perceived restorativeness. We found that a virtual walk through a pine forest was capable of improving psychological well-being and invoking feelings of restorativeness, with walks through different environments having some similar and some different effects. The results presented here highlight the importance of examining within-category differences between multiple stimuli that might be labeled as "natural" or "urban" even when environments are comparable on metrics such as amount of green space. Future research should consider each environment in terms of its own properties (e.g., fascination, perceptual complexity, low-level visual and acoustic features) rather than in a dichotomized way, as this could aid in our understanding and appreciation of the apparent psychological benefits of interacting with nature. 


\section{References}

Asim, F., Rai, S., \& Shree, V. (2020). Biophilic Architecture for restoration and therapy within the built environment. Visions for Sustainability, 15, 53-79. https://doi.org/10.13135/2384$8677 / 5104$

Basu, A., Duvall, J., \& Kaplan, R. (2019). Attention restoration theory: Exploring the role of soft fascination and mental bandwidth. Environment and Behavior, 51(9-10), 1055-1081. https://doi.org/10.1177/0013916518774400

Benjamini, Y., \& Hochberg, Y. (1995). Controlling the false discovery rate: A practical and powerful approach to multiple testing. Journal of the Royal Statistical Society: Series B (Methodological), 57(1), 289-300. https://doi.org/10.1111/j.2517-6161.1995.tb02031.x

Berman, M. G., Hout, M. C., Kardan, O., Hunter, M. R., Yourganov, G., Henderson, J. M., Hanayik, T., Karimi, H., \& Jonides, J. (2014). The perception of naturalness correlates with low-level visual features of environmental scenes. PLOS ONE, 9(12), e114572. https://doi.org/10.1371/journal.pone.0114572

Berman, M. G., Jonides, J., \& Kaplan, S. (2008). The cognitive benefits of interacting with nature. Psychological Science, 19(12), 1207-1212. https://doi.org/10.1111/j.14679280.2008.02225.x

Berman, M. G., Kross, E., Krpan, K. M., Askren, M. K., Burson, A., Deldin, P. J., Kaplan, S., Sherdell, L., Gotlib, I. H., \& Jonides, J. (2012). Interacting with nature improves cognition and affect for individuals with depression. Journal of Affective Disorders, 140(3), 300305. https://doi.org/10.1016/j.jad.2012.03.012

Berman, M. G., Stier, A. J., \& Akcelik, G. N. (2019). Environmental neuroscience. The American Psychologist, 74(9), 1039-1052. https://doi.org/10.1037/amp0000583

Bratman, G. N., Daily, G. C., Levy, B. J., \& Gross, J. J. (2015). The benefits of nature experience: Improved affect and cognition. Landscape and Urban Planning, 138, 41-50. https://doi.org/10.1016/j.landurbplan.2015.02.005 
Brown, D. K., Barton, J. L., \& Gladwell, V. F. (2013). Viewing nature scenes positively affects recovery of autonomic function following acute-mental stress. Environmental Science \& Technology, 47(11), 5562-5569. https://doi.org/10.1021/es305019p

Browning, M. H. E. M., Saeidi-Rizi, F., McAnirlin, O., Yoon, H., \& Pei, Y. (2021). The role of methodological choices in the effects of experimental exposure to simulated natural landscapes on human health and cognitive performance: A systematic review. Environment and Behavior, 53(7), 687-731. https://doi.org/10.1177/0013916520906481

Brymer, D. E., Cuddihy, T. F., \& Sharma-Brymer, V. (2010). The role of nature-based experiences in the development and maintenance of wellness. Asia-Pacific Journal of Health, Sport and Physical Education, 1(2), 21-27.

https://doi.org/10.1080/18377122.2010.9730328

Capaldi, C. A., Dopko, R. L., \& Zelenski, J. M. (2014). The relationship between nature connectedness and happiness: A meta-analysis. Frontiers in Psychology, 5. https://doi.org/10.3389/fpsyg.2014.00976

Capaldi, C. A., Passmore, H.-A., Nisbet, E. K., Zelenski, J. M., \& Dopko, R. L. (2015). Flourishing in nature: A review of the benefits of connecting with nature and its application as a wellbeing intervention. International Journal of Wellbeing, 5(4), Article 4. https://doi.org/10.5502/ijw.v5i4.449

Coburn, A., Kardan, O., Kotabe, H., Steinberg, J., Hout, M. C., Robbins, A., MacDonald, J., Hayn-Leichsenring, G., \& Berman, M. G. (2019). Psychological responses to natural patterns in architecture. Journal of Environmental Psychology, 62, 133-145. https://doi.org/10.1016/j.jenvp.2019.02.007

Dadvand, P., Nieuwenhuijsen, M. J., Esnaola, M., Forns, J., Basagaña, X., Alvarez-Pedrerol, M., Rivas, I., López-Vicente, M., Pascual, M. D. C., Su, J., Jerrett, M., Querol, X., \& Sunyer, J. (2015). Green spaces and cognitive development in primary schoolchildren. 
Proceedings of the National Academy of Sciences, 112(26), 7937-7942. https://doi.org/10.1073/pnas. 1503402112

de Leeuw, J. R. (2015). jsPsych: A JavaScript library for creating behavioral experiments in a Web browser. Behavior Research Methods, 47(1), 1-12. https://doi.org/10.3758/s13428014-0458-y

Engemann, K., Pedersen, C. B., Arge, L., Tsirogiannis, C., Mortensen, P. B., \& Svenning, J.-C. (2019). Residential green space in childhood is associated with lower risk of psychiatric disorders from adolescence into adulthood. Proceedings of the National Academy of Sciences, 116(11), 5188-5193. https://doi.org/10.1073/pnas.1807504116

Faul, F., Erdfelder, E., Buchner, A., \& Lang, A.-G. (2009). Statistical power analyses using G*Power 3.1: Tests for correlation and regression analyses. Behavior Research Methods, 41(4), 1149-1160. https://doi.org/10.3758/BRM.41.4.1149

Felsten, G. (2009). Where to take a study break on the college campus: An attention restoration theory perspective. Journal of Environmental Psychology, 29(1), 160-167. https://doi.org/10.1016/j.jenvp.2008.11.006

Gosling, S. D., Rentfrow, P. J., \& Swann, W. B. (2003). A very brief measure of the Big-Five personality domains. Journal of Research in Personality, 37(6), 504-528. https://doi.org/10.1016/S0092-6566(03)00046-1

Hughes, M. E., Waite, L. J., Hawkley, L. C., \& Cacioppo, J. T. (2004). A short scale for measuring loneliness in large surveys: Results from two population-based studies. Research on Aging, 26(6), 655-672. https://doi.org/10.1177/0164027504268574 Kaplan, S. (1995). The restorative benefits of nature: Toward an integrative framework. Journal of Environmental Psychology, 15(3), 169-182. https://doi.org/10.1016/02724944(95)90001-2 
Kaplan, S., \& Berman, M. G. (2010). Directed attention as a common resource for executive functioning and self-regulation. Perspectives on Psychological Science, 5(1), 43-57. https://doi.org/10.1177/1745691609356784

Kardan, O., Gozdyra, P., Misic, B., Moola, F., Palmer, L. J., Paus, T., \& Berman, M. G. (2015). Neighborhood greenspace and health in a large urban center. Scientific Reports, 5, 11610. https://doi.org/10.1038/srep11610

Kellert, S. R., \& Wilson, E. O. (1993). The Biophilia Hypothesis. Island Press.

Litman, L., Robinson, J., \& Abberbock, T. (2017). TurkPrime.com: A versatile crowdsourcing data acquisition platform for the behavioral sciences. Behavior Research Methods, 49(2), 433-442. https://doi.org/10.3758/s13428-016-0727-z

Martin, L., White, M. P., Hunt, A., Richardson, M., Pahl, S., \& Burt, J. (2020). Nature contact, nature connectedness and associations with health, wellbeing and pro-environmental behaviours. Journal of Environmental Psychology, 68, 101389.

https://doi.org/10.1016/j.jenvp.2020.101389

McAllister, E., Bhullar, N., \& Schutte, N. S. (2017). Into the woods or a stroll in the park: How virtual contact with nature impacts positive and negative affect. International Journal of Environmental Research and Public Health, 14(7), 786.

https://doi.org/10.3390/ijerph14070786

McMahan, E. A., \& Estes, D. (2015). The effect of contact with natural environments on positive and negative affect: A meta-analysis. The Journal of Positive Psychology, 10(6), 507519. https://doi.org/10.1080/17439760.2014.994224

McMillan, R., Kaufman, S. B., \& Singer, J. L. (2013). Ode to positive constructive daydreaming. Frontiers in Psychology, 0. https://doi.org/10.3389/fpsyg.2013.00626

Meidenbauer, K. L., Stenfors, C. U. D., Bratman, G. N., Gross, J. J., Schertz, K. E., Choe, K. W., \& Berman, M. G. (2020). The affective benefits of nature exposure: What's nature 
got to do with it? Journal of Environmental Psychology, 72, 101498. https://doi.org/10.1016/j.jenvp.2020.101498

Meidenbauer, K. L., Stenfors, C. U. D., Young, J., Layden, E. A., Schertz, K. E., Kardan, O., Decety, J., \& Berman, M. G. (2019). The gradual development of the preference for natural environments. Journal of Environmental Psychology, 65, 101328. https://doi.org/10.1016/j.jenvp.2019.101328

Nisbet, E. K., Zelenski, J. M., \& Murphy, S. A. (2009). The nature relatedness scale: Linking individuals' connection with nature to environmental concern and behavior. Environment and Behavior, 41(5), 715-740. https://doi.org/10.1177/0013916508318748

Norling, J. C., Sibthorp, J., \& Ruddell, E. (2008). Perceived Restorativeness for Activities Scale (PRAS): Development and validation. Journal of Physical Activity and Health, 5(1), 184195. https://doi.org/10.1123/jpah.5.1.184

Schertz, K. E., \& Berman, M. G. (2019). Understanding nature and its cognitive benefits. Current Directions in Psychological Science, 28(5), 496-502. https://doi.org/10.1177/0963721419854100

Schertz, K. E., Sachdeva, S., Kardan, O., Kotabe, H. P., Wolf, K. L., \& Berman, M. G. (2018). A thought in the park: The influence of naturalness and low-level visual features on expressed thoughts. Cognition, 174, 82-93. https://doi.org/10.1016/j.cognition.2018.01.011

Schertz, K. E., Saxon, J., Cardenas-Iniguez, C., Bettencourt, L. M. A., Ding, Y., Hoffmann, H., \& Berman, M. G. (2021). Neighborhood street activity and greenspace usage uniquely contribute to predicting crime. Npj Urban Sustainability, 1(1), 1-10. https://doi.org/10.1038/s42949-020-00005-7

Southon, G. E., Jorgensen, A., Dunnett, N., Hoyle, H., \& Evans, K. L. (2018). Perceived species-richness in urban green spaces: Cues, accuracy and well-being impacts. 
Landscape and Urban Planning, 172, 1-10.

https://doi.org/10.1016/j.landurbplan.2017.12.002

Spielberger, C. D. (1983). State-Trait Anxiety Inventory for Adults (STAI-AD). APA PsychTests. https://doi.org/10.1037/t06496-000

Stenfors, C. U. D., Van Hedger, S. C., Schertz, K. E., Meyer, F. A. C., Smith, K. E. L., Norman, G. J., Bourrier, S. C., Enns, J. T., Kardan, O., Jonides, J., \& Berman, M. G. (2019). Positive effects of nature on cognitive performance across multiple experiments: Test order but not affect modulates the cognitive effects. Frontiers in Psychology, 10. https://doi.org/10.3389/fpsyg.2019.01413

Stevenson, M. P., Schilhab, T., \& Bentsen, P. (2018). Attention Restoration Theory II: A systematic review to clarify attention processes affected by exposure to natural environments. Journal of Toxicology and Environmental Health, Part B, 21(4), 227-268. https://doi.org/10.1080/10937404.2018.1505571

Ulrich, R. S. (1981). Natural versus urban scenes: Some psychophysiological effects. Environment and Behavior, 13(5), 523-556. https://doi.org/10.1177/0013916581135001

Ulrich, R. S. (1983). Aesthetic and affective response to natural environment. In I. Altman \& J. F. Wohlwill (Eds.), Behavior and the Natural Environment (pp. 85-125). Springer US. https://doi.org/10.1007/978-1-4613-3539-9_4

Ulrich, R. S. (1984). View through a window may influence recovery from surgery. Science, 224(4647), 420-421. https://doi.org/10.1126/science.6143402

Ulrich, R. S., Simons, R. F., Losito, B. D., Fiorito, E., Miles, M. A., \& Zelson, M. (1991). Stress recovery during exposure to natural and urban environments. Journal of Environmental Psychology, 11(3), 201-230. https://doi.org/10.1016/S0272-4944(05)80184-7

Van Hedger, S. C., Nusbaum, H. C., Clohisy, L., Jaeggi, S. M., Buschkuehl, M., \& Berman, M. G. (2019). Of cricket chirps and car horns: The effect of nature sounds on cognitive 
performance. Psychonomic Bulletin \& Review, 26(2), 522-530. https://doi.org/10.3758/s13423-018-1539-1

Van Hedger, S. C., Nusbaum, H. C., Heald, S. L. M., Huang, A., Kotabe, H. P., \& Berman, M. G. (2019). The Aesthetic Preference for Nature Sounds Depends on Sound Object Recognition. Cognitive Science, 43(5). https://doi.org/10.1111/cogs.12734

Watson, D., Clark, L. A., \& Tellegen, A. (1988). Development and validation of brief measures of positive and negative affect: The PANAS scales. Journal of Personality and Social Psychology, 54(6), 1063-1070. https://doi.org/10.1037/0022-3514.54.6.1063

White, M., Smith, A., Humphryes, K., Pahl, S., Snelling, D., \& Depledge, M. (2010). Blue space: The importance of water for preference, affect, and restorativeness ratings of natural and built scenes. Journal of Environmental Psychology, 30(4), 482-493. https://doi.org/10.1016/j.jenvp.2010.04.004

Wilkie, S., Thompson, E., Cranner, P., \& Ginty, K. (2020). Attention restoration theory as a framework for analysis of Tweets about urban green space: A case study. Landscape Research, 45(6), 777-788. https://doi.org/10.1080/01426397.2020.1738363

Wilson, E. O. (1984). Biophilia. Harvard University Press.

Wood, E., Harsant, A., Dallimer, M., Cronin de Chavez, A., McEachan, R. R. C., \& Hassall, C. (2018). Not all green space is created equal: Biodiversity predicts psychological restorative benefits from urban green space. Frontiers in Psychology, 9. https://doi.org/10.3389/fpsyg.2018.02320

Wyles, K. J., White, M. P., Hattam, C., Pahl, S., King, H., \& Austen, M. (2019). Are some natural environments more psychologically beneficial than others? The importance of type and quality on connectedness to nature and psychological restoration. Environment and Behavior, 51(2), 111-143. https://doi.org/10.1177/0013916517738312 


\section{Tables and Figures}

Table 1: Mean trait measures across virtual walk conditions

\begin{tabular}{lcccccc}
\hline Measure & $\begin{array}{c}\text { Pine } \\
\text { Forest }\end{array}$ & $\begin{array}{c}\text { Farmed } \\
\text { Field }\end{array}$ & $\begin{array}{c}\text { Tree-lined } \\
\text { Neighborhood }\end{array}$ & $\begin{array}{c}\text { Bustling } \\
\text { City }\end{array}$ & $F$ & $\eta^{2}{ }_{p}$ \\
\hline TIPI - O & $4.77(1.78)$ & $5.04(1.60)$ & $5.44(1.20)$ & $5.49(1.46)$ & 2.20 & 0.037 \\
TIPI - C & $4.62(1.22)$ & $4.73(1.31)$ & $4.90(1.16)$ & $4.82(1.29)$ & 0.40 & 0.007 \\
TIPI - E & $3.18(2.02)$ & $3.37(1.98)$ & $2.89(1.89)$ & $3.88(2.05)$ & 1.87 & 0.031 \\
TIPI - A & $5.36(1.56)$ & $5.72(1.22)$ & $5.65(1.29)$ & $5.49(1.22)$ & 0.68 & 0.012 \\
TIPI - ES & $4.92(1.85)$ & $5.10(1.80)$ & $5.21(1.52)$ & $5.21(1.80)$ & 0.86 & 0.004 \\
STAI - T & $2.04(0.83)$ & $1.97(0.71)$ & $1.87(0.65)$ & $1.80(0.66)$ & 0.91 & 0.015 \\
UCLA - L & $2.34(1.17)$ & $2.02(0.83)$ & $2.20(0.91)$ & $2.00(1.00)$ & 1.20 & 0.020 \\
NRS & $3.53(0.78)$ & $3.71(0.78)$ & $3.66(0.78)$ & $3.45(0.79)$ & 0.36 & 0.018 \\
\hline
\end{tabular}

Note: $T I P I=$ Ten-Item Personality Inventory; $O=$ Openness, $C=$ Conscientiousness, $E=$ Extraversion; $A$ = Agreeableness; $E S$ = Emotional Stability; NRS = Nature Relatedness Scale; STAI - T = State-Trait Anxiety Inventory, Trait; ULS-8 = UCLA Loneliness Scale. Standard deviations are printed in parentheses. 
Figure 1: Summary of the experimental design

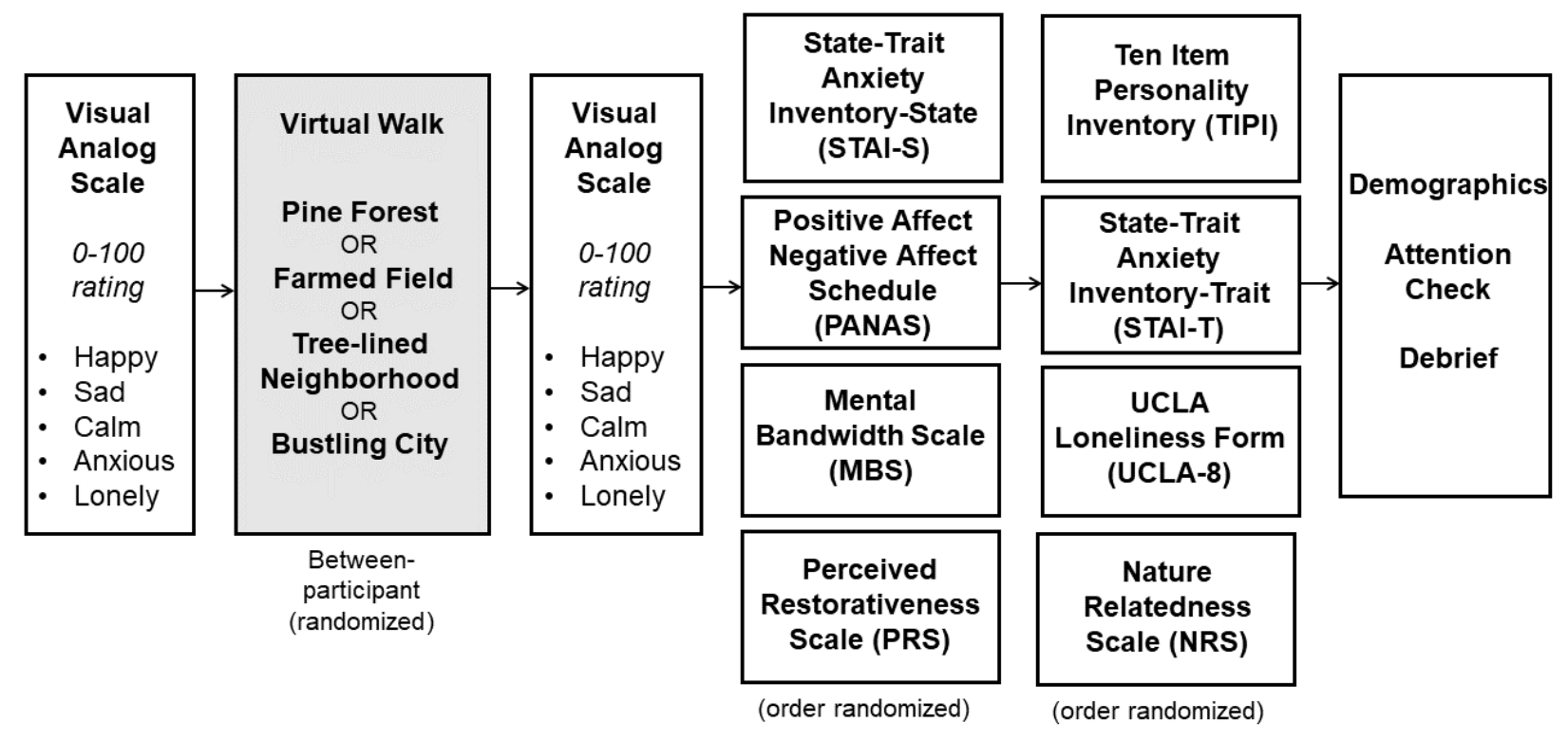


Figure 2: Still images from each of the virtual walks

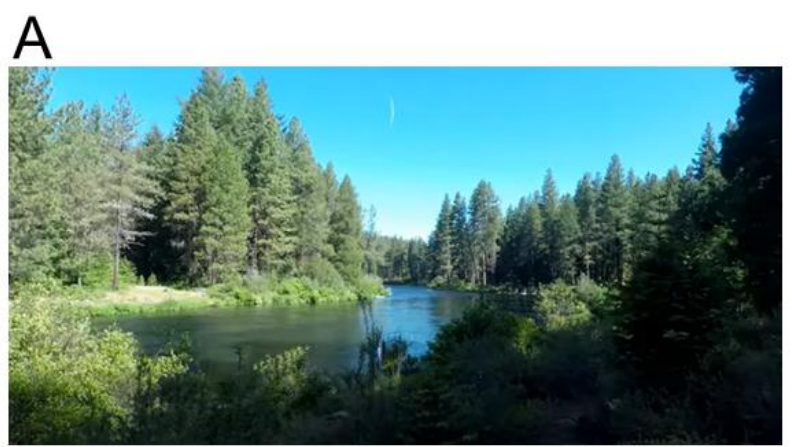

B
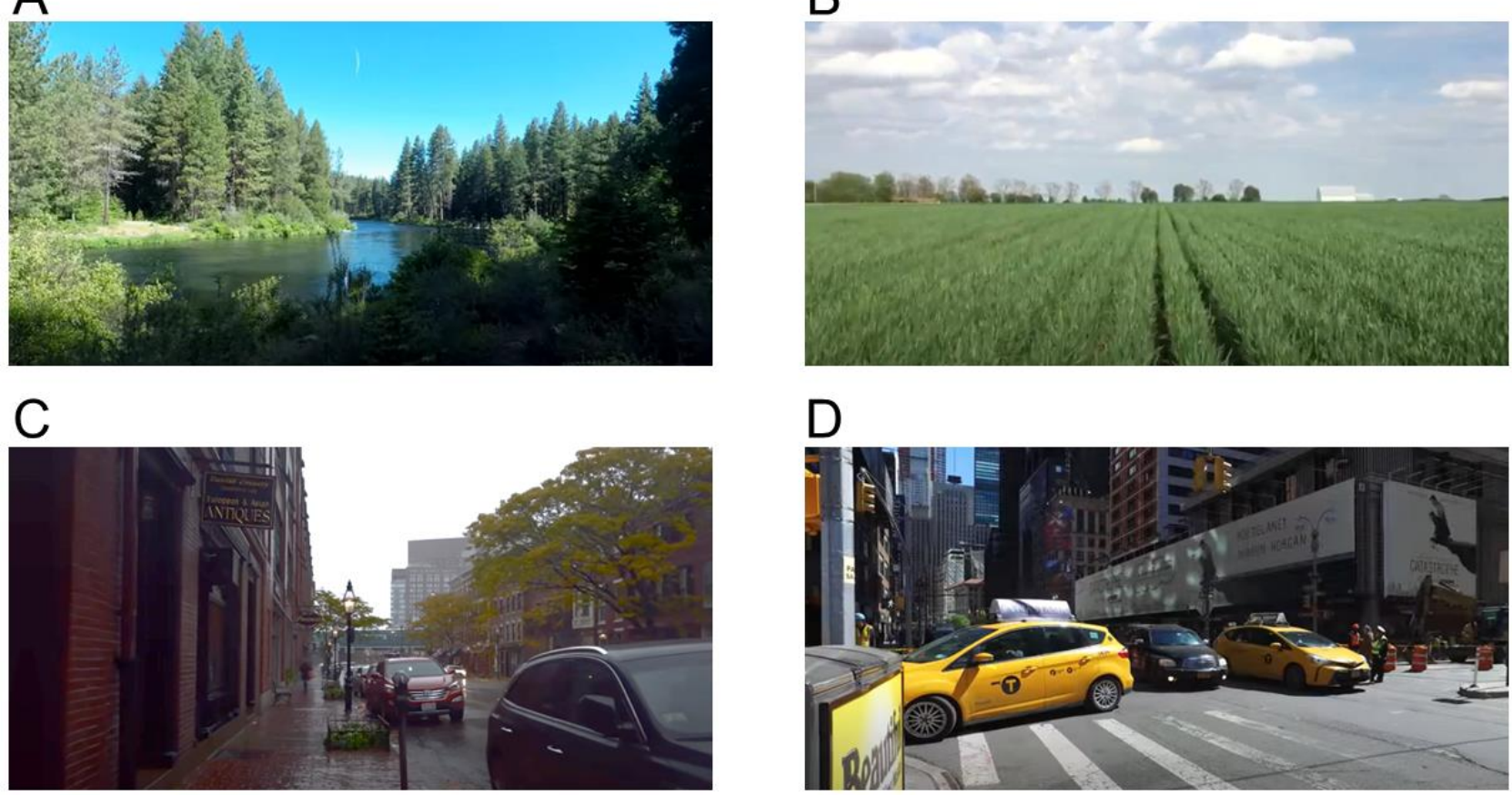

Note: Panel A depicts the pine forest walk, Panel B depicts the farmed field walk, Panel C depicts the tree-lined neighborhood walk, and Panel $D$ depicts the bustling city center walk 
Figure 3: Mood ratings as a function of virtual walk condition
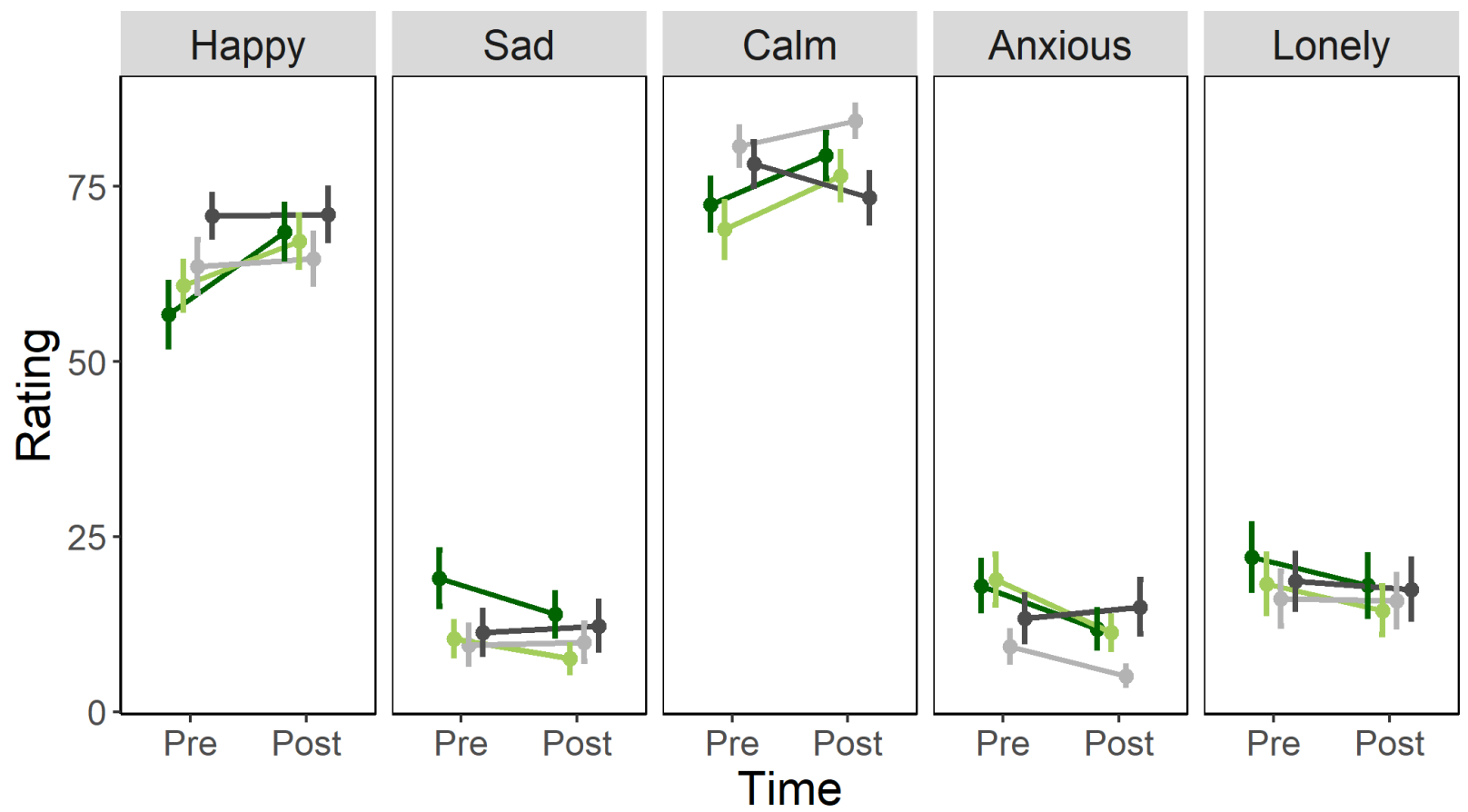

$\multimap$ Pine Forest $\leadsto-$ Farmed Field $\approx-$ Tree-lined Neighborhood $\curvearrowleft$ Bustling City

Note: Ratings were made on a 100-point visual analog scale immediately before and after the virtual walk. Error bars represent \pm 1 standard error of the mean. 
Figure 4: Judgments of restorativeness as a function of virtual walk condition
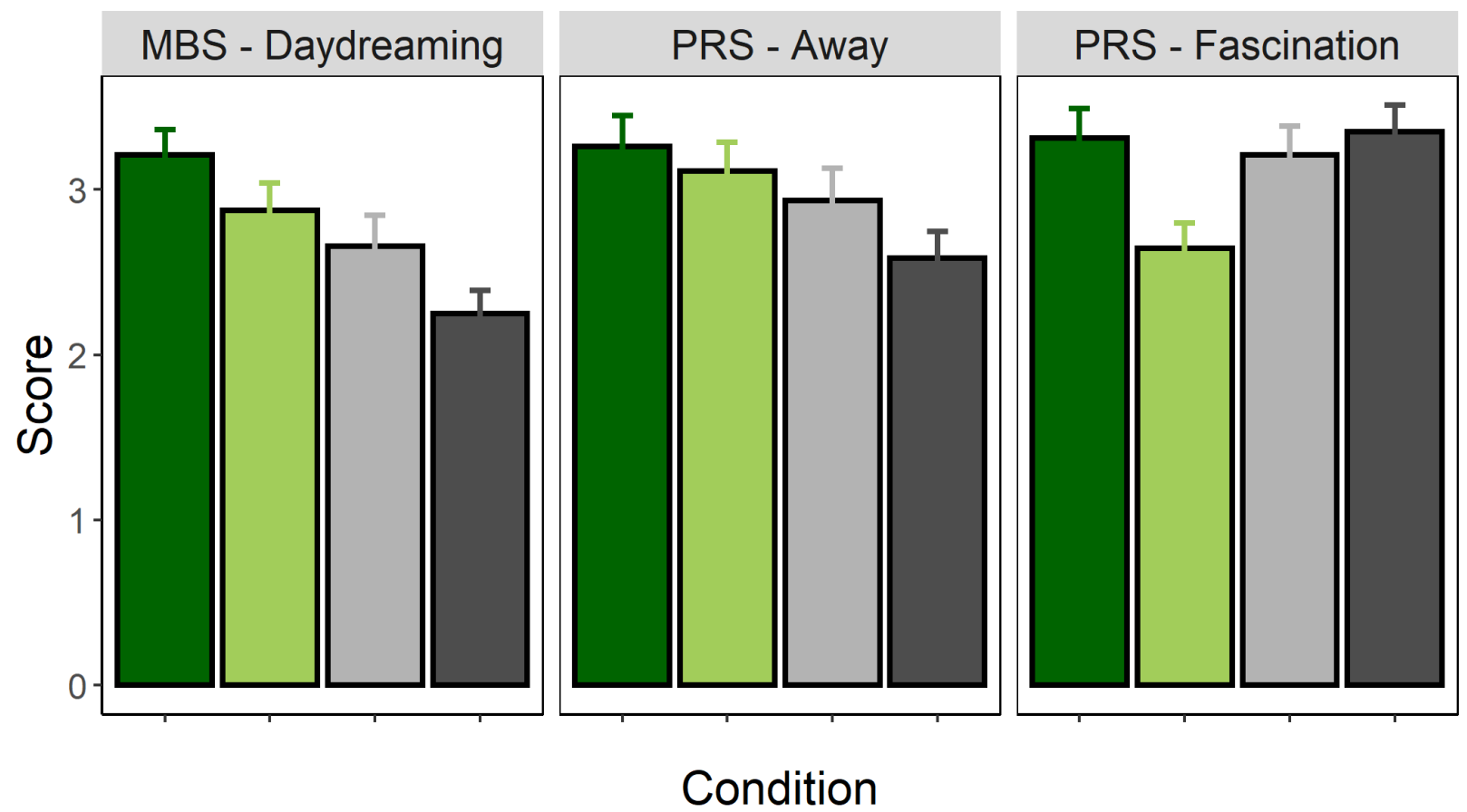

Condition

$\square$ Pine Forest $\square$ Farmed Field $\square$ Tree-lined Neighborhood $\square$ Bustling City

Note: MBS = Mental Bandwidth Scale; PRS = Perceived Restorativeness Scale. Error bars represent \pm 1 standard error of the mean. 